\title{
BIOLOGÍA Y APROVECHAMIENTO
}

\author{
de Agave leChuguilla Torrey
}

\author{
Juan Antonio Reyes-Agüero ${ }^{1 *}$ Juan Rogelio Aguirre-Rivera ${ }^{1}$ \\ Cecilia Beatriz Peña-Valdivia ${ }^{2}$
}

\begin{abstract}
•Autor para la correspondencia. Instituto de Investigación de Zonas Desérticas de la Universidad Autónoma de San Luis Potosí. Altair 200. Fracc. del Llano. 78170 San Luis Potosí, S.L.P.

Tel. (014) 822 2130, Fax (014) 822 2718. Correo electrónico: reyesaguero@yahoo.com

${ }^{2}$ Especialidad de Botánica, Instituto de Recursos Naturales, Colegio de Postgraduados. km. 35.5 Carr. México-Texcoco.

Correo electrónico: cecilia@colpos.colpos.mx
\end{abstract}

\begin{abstract}
Resumen: La lechuguilla (Agave lechuguilla) es una planta de las zonas áridas y semiáridas de México y sur de Estados Unidos, utilizada por su fibra desde hace por lo menos unos 8000 años; actualmente es fundamental para la economía de unos 20000 recolectores y sus familias. A. lechuguilla es una planta monocárpica polianual, con alta capacidad de competencia intra e interespecífica, amplia resistencia al ataque de plagas, a la herbivoría y a condiciones ambientales extremas, productividad alta $\left(0.38 \mathrm{~kg}\right.$ de biomasa seca $\left./ \mathrm{m}^{2} / \mathrm{año}\right)$, distribución geográfica amplia (unos $100000 \mathrm{~km}^{2}$ ) y capacidad reproductiva vegetativa y sexual elevada. El aprovechamiento persistente de la lechuguilla y su mejoramiento requiere del manejo fundamentado de las poblaciones silvestres, más que la aplicación de prácticas típicas de la agronomía.

Palabras clave: fibras duras, ixtle, recolección, plantas útiles, zonas áridas.

Abstract: The lechuguilla (Agave lechuguilla) is a native plant of arid and semiarid regions from Mexico and south of United States; it has been used for its fiber since approximately 8000 years ago; today, it is fundamental for economy of aproximately 20000 peasants and their families. A. lechuguilla is a monocarpic plant, with high capacity of intra and inter-specific competence, high resistance to pests, herbivory and environmental strong conditions, its productivity is high $\left(0.38 \mathrm{~kg} / \mathrm{m}^{2} /\right.$ year $)$, it has broad geographic distribution (aproximatly $100000 \mathrm{~km}^{2}$ ) and high capacity of vegetative and sexual reproduction. The continuous exploitation of lechuguilla and its improvement require a scientific management of wild populations, instead the use of typical agronomical practices.
\end{abstract}

Key words: arid lands, gathering, hard fiber, ixtle, useful plants.

U na de las principales actividades económicas de los pobladores actuales de las zonas áridas y semiáridas del altiplano mexicano, es la recolección de materiales naturales para autoconsumo y venta (Aguirre, 1983). Se ha documentado que tan sólo para autoconsumo, se recolectan 95 especies de plantas en los tres tipos de matorrales característicos de dichas zonas (Juárez et al., 1996). Entre las especies más recolectadas para su venta están la lechuguilla (Agave lechuguilla), la palma samandoca (Yucca carnerosana), la candelilla (Euphorbia antisiphylitica), el mezquite (Prosopis spp.) y los nopales (Opuntia spp.). En este grupo destaca la lechuguilla porque se ha recolectado intensa y continuamente desde el siglo XIX. Actual- mente la lechuguilla es un elemento fundamental de la economía de numerosas familias campesinas, por los ingresos netos que representa, al menos durante un tercio del año; la fibra de lechuguilla es la materia prima para diversas empresas nacionales y extranjeras, que la utilizan en la elaboración de diferentes productos.

Desde el punto de vista biológico, la lechuguilla es excepcional, ya que es una de las dos especies de Agave con la distribución natural más amplia y las mayores densidades en América del Norte, donde llega a ser dominante fisonómico del matorral desértico rosetófilo y su productividad es superior a la media de las especies de su ambiente. 
El objetivo del presente trabajo fue reunir y revisar la información publicada sobre Agave lechuguilla, para establecer el estado actual del conocimiento sobre su biología y aprovechamiento, particularmente el manejo y conservación de sus poblaciones naturales, su utilidad como planta modelo en estudios fisiológicos, bioquímicos y anatómicos y sus usos diferentes a los tradicionales.

\section{Nomenclatura tradicional y científica}

El nombre tradicional y el epíteto específico de Agave lechuguilla significan lechuga pequeña (Dewey 1965; McCleary 1955). En efecto, los pobladores rurales de algunas zonas áridas y semiáridas del norte de México utilizan los sufijos illo e illa, en los diminutivos, y la semejanza con la lechuga fue establecida por los conquistadores españoles. En las zonas lechuguilleras, los campesinos distinguen $A$. lechuguilla de otras lechuguillas, como son la lechuguilla mansa (A. filifera) (Reyes, 1992) y la lechuguilla de Jaumave ( $A$. funkiana) (Dewey, 1965; Marroquín et al., 1981; Ramírez, 1932). A la vez, A. lechuguilla es conocida también como maguey del cerro o pita; en otomí se le llama tzeth, tzuta o ratzitá, en tarahumara sóko y en náhuatl metometl (Aguilar y Zolla, 1982; Martínez, 1979; Nieto, 1983; Pennington, 1957; Ramírez, 1932). Metometl proviene de metolin, tiña o corrosión y de metl, maguey (Simeón, 1977).

Charles Wright, de la Comisión Binacional de Límites entre México y Estados Unidos, recolectó una lechuguilla en 1849; diez años después, el taxónomo J. Torrey la denominó Agave lecheguilla, y él mismo señaló que el nombre común era lechuguilla (McCleary, 1955). Posteriormente, Mulford (1896, citado por McCleary, 1955) estableció que en la grafía de la palabra lechuguilla había un error tipográfico cometido por J. Torrey. Sin embargo, varios autores (Bailey y Bailey, 1976; Correll y Jhonston, 1970; Ramírez, 1932; Sheldon, 1980; Standley, 1920-1926) continuaron usando el epíteto lecheguilla. McCleary (1955), con base en el Código de Nomenclatura Botánica, corrigió formalmente el error y Gentry (1982) confirmó dicha corrección.

La sinonimia científica registrada por Gentry (1982) y Magallán (1998) para Agave lechuguilla es:

A. poselgeri Salm-Dyck, Bonplandia 7:92, 1859.

A. lophantha Schiede var. poselgeri (Salm-Dyck) A. Berger, Agaven 93-94, 1915.

A. lophantha Schiede var. tamaulipasana A. Berger, Agaven 94, 1915.

A. multilineata Baker, Handb. Amaryllideae 168, 1888.

A. heteracantha Hort., non Zucc., 1833, nec Baker, 1877.

\section{Morfología}

Agave lechuguilla es un arbusto rosetófilo, acaule, pequeño, con 11 a 30 hojas en promedio (cuadro 1); puede medir entre 30 y $40 \mathrm{~cm}$ de ancho y de 20 a 70 $\mathrm{cm}$ de alto (Dewey, 1965; Freeman y Reid, 1985; Gentry, 1982; Magallán, 1998; Rzedowski, 1964; Sheldon, 1980). Sus raíces son largas, fibrosas, delgadas y se distribuyen a una profundidad entre 8 y $12 \mathrm{~cm}$ del suelo (Nobel y Quero, 1986). Sus hojas se forman a partir de una yema apical considerablemente desarrollada (denominada cogollo) (Font Quer, 1953), que en Agave es un cono formado por hojas jóvenes enrolladas sobre un eje central; las hojas jóvenes recién desplegadas son verde-blanquecinas o verde-azuladas (Dewey, 1965; Rzedowski, 1964; Sheldon, 1980; Zapién, 1981). Las hojas maduras miden entre 25 y $50 \mathrm{~cm}$ de alto y 2.5 a $6 \mathrm{~cm}$ de ancho, tienen forma lineal lanceolada, con un color que va del verde claro al amarillo verdoso, son entre difusas y erectas, algunas veces incurvadas y raramente falcadas, cóncavas en el haz y convexas en el envés; algunas veces presentan una franja longitudinal de un color verde más claro en el haz, y franjas estrechas, verde-oscuras, de entre 30 y $60 \mathrm{~cm}$ de largo en el envés. El margen de las hojas es entero, castaño claro a gris, se separa fácilmente en las hojas secas, y presenta 8 a 20 dientes (espinas) deflejos por lado; dichos dientes miden de 2 a $7 \mathrm{~mm}$, de color castaño o más frecuentemente gris claro, y separados de $1.5 \mathrm{a} 4 \mathrm{~cm}$. El ápice es una espina fuerte, cónica, subulada, grisácea de 1.5 a $4 \mathrm{~cm}$ (Berlanga et al., 1992a; Dewey, 1965; Gentry, 1982; Sheldon, 1980). La inflorescencia recibe los nombres de quiote, mequiote (del náhuatl metl, maguey y quiyotl o quiotl, vástago o tallo), garrocha o bohordo; es un escapo espigado de 2.5 a 3.6 o hasta $6 \mathrm{~m}$ de alto, con la superficie glauca (Berlanga et al., 1992a; Dewey, 1965; Flores, 1986; Gentry, 1982; Magallán, 1998; Rzedowski, 1964; Sheldon, 1980) y brácteas deltoides, de $10-20 \mathrm{~cm}$ de largo, con espina en

Cuadro 1. Número de hojas de Agave lechuguilla en una población de El Paso, Texas.

\begin{tabular}{cc}
\hline Número de hojas & Porcentaje de la población \\
\hline $1-10$ & 12 \\
$11-20$ & 38 \\
$21-30$ & 36 \\
$31-40$ & 8 \\
$41-50$ & 4 \\
$51-60$ & 2 \\
\hline
\end{tabular}

(Fuente: Freeman y Reid, 1985) 
el ápice (Magallán, 1998). Las flores tienen pedicelos de 20 a $50 \mathrm{~mm}$ y se agrupan en pares o triadas. El ovario es ínfero, trilocular, de 15 a $22 \mathrm{~mm}$ de largo, fusiforme, redondeadamente angulado y constricto en el cuello, óvulos anátropos con placentación axial, estilo de 33 a $47 \mathrm{~mm}$ y estigma de 1.5 a $3.5 \mathrm{~mm}$; el perianto lo conforman seis tépalos de 13 a $20 \mathrm{~mm}$, amarillos, frecuentemente con manchas púrpuras lineales, ascendentes, involutos alrededor de los filamentos, los externos anchamante imbricados, protegidos por brácteas verde-amarillas o rojizas subiguales; seis filamentos de 25 a $40 \mathrm{~cm}$ de largo, extendidos; anteras de 15 a $20 \mathrm{~cm}$ de longitud. El fruto es castaño a negro, capsular, oblongo a piriforme, de 1.5 a $2.5 \mathrm{~cm}$ de longitud por $1.0 \mathrm{a} 1.8 \mathrm{~cm}$ de ancho, es sésil o con pedicelos muy cortos, redondeado, con un rostro (pico) pequeño en el ápice, y glauco. Las semillas por fruto son numerosas, planas y brillantes, de 4.5 a 6 $\mathrm{mm}$ de longitud por 3.5 a $4.5 \mathrm{~mm}$ de ancho, con hilo pequeño y costilludo, y alas alrededor del lado curvo (Berlanga et al., 1992a; Flores, 1986; Gentry, 1982; Grove, 1941). El número cromosómico de la lechuguilla es $2 \mathrm{n}=90$ (Flores, 1986).

Existen indicios sobre la existencia de variantes de A. lechuguilla (Rzedowski, 1964). Al respecto, Martínez (1936) afirmó, sin precisar medidas, que la lechuguilla del sur de Coahuila es varias veces menor que la de San Luis Potosí; en efecto, la lechuguilla de Texas mide entre 25 y $40 \mathrm{~cm}$ (Correll y Johnston, 1970) y la de Querétaro alcanza hasta $70 \mathrm{~cm}$ de altura (Magallán, 1998). Por su parte, Quero y Nobel (1987) reconocieron tres poblaciones localizadas al sureste de Saltillo, Coahuila, en altitudes de 1000,1400 y $1900 \mathrm{~m}$, cuyo número medio de hojas fue de 22, 36 y 47, respectivamente. Silva et al. (1998) y Eguiarte y Silva (2000) registraron que las flores de las lechuguillas del extremo sur de su distribución, son verdosas, claras, glaucas y largas y en el norte son rojas, más anchas, menos largas y producen menos néctar; además, las lechuguillas del norte tienen menos flores por inflorescencia y por lo tanto, producen menos frutos.

\section{Ecofisiología}

Nobel y Quero (1986) evaluaron características ecofisiológicas de algunas poblaciones naturales de $A$. lechuguilla de Saltillo, Coah. Estos autores encontraron que en las plantas con menos de diez hojas, la biomasa seca total está asignada homogéneamente entre hojas (vivas y muertas), cogollo, tallo y raíz. En contraste, en plantas con más de 20 hojas, la mayor parte de la biomasa (60\%) está en las hojas, y la menor $(4 \%)$ en las raíces. En promedio, durante la vida de la planta, el cogollo contiene aproximadamente $10 \%$ de la biomasa total, sólo cuando la planta llega a tener 60 hojas, dicha proporción disminuye hasta el $5 \%$. Algo semejante ocurre con el tallo, pues regularmente contiene el $20 \%$ del peso, pero en plantas grandes su biomasa sólo representa el $12 \%$. El número de hojas por planta es muy variable; así, Freeman y Reid (1985), en una comunidad cercana a El Paso, Texas reconocieron seis clases de tamaño de planta; el mayor porcentaje de individuos tuvo entre 11 y 30 hojas (cuadro 1 ). De la radiación fotosintéticamente activa que se recibe en un día $\left(42.2 \mathrm{~mol} / \mathrm{m}^{2}\right)$, las hojas del cogollo reciben $45 \%\left(19.1 \mathrm{~mol} / \mathrm{m}^{2} /\right.$ día $)$ y las hojas medias e inferiores $43 \%$ ( $18.3 \mathrm{~mol} / \mathrm{m}^{2} /$ día) (Nobel y Quero, 1986). Eickmeier y Adams (1978) comprobaron que $A$. lechuguilla es una planta con metabolismo fotosintético tipo MAC, ya que en su ambiente natural la mayor absorción de $\mathrm{CO}_{2}$ la realiza durante la noche. Por su parte, Nobel y Quero (1986) determinaron que después de siete días con suspensión de riego $\left(\psi_{\text {suelo }}<-0.5 \mathrm{Mpa}\right)$ la asimilación neta de $\mathrm{CO}_{2}$ por A. lechuguilla, en un período de $24 \mathrm{~h}$, fue de 100 mmoles $/ \mathrm{m}^{2}$; sin embargo, con riego adecuado $\left(\psi_{\text {sueto }}\right.$ $>-0.5 \mathrm{Mpa}$ ) dicha asimilación se incrementó a 172 mmoles $/ \mathrm{m}^{2}$. En estas condiciones de riego, sólo $85 \%$ de la asimilación correspondió al período nocturno, y como no se observó el paso completo de metabolismo fotosintético tipo MAC a tipo $\mathrm{C}_{3}$, sólo puede señalarse que $A$. lechuguilla no es una planta MAC estricta. Durante su adaptación a los períodos secos, la conductancia estomática disminuye de $2.3 \mathrm{~mm} / \mathrm{s}$ (con humedad adecuada) a 1.7 y $0.11 \mathrm{~mm} / \mathrm{s}$, después de siete y 13 días sin aporte de agua, respectivamente (Nobel y Quero, 1986). En un ambiente con $375 \mathrm{~mm}$ de precipitación, la productividad total de biomasa es de $0.38 \mathrm{~kg} / \mathrm{m}^{2} / \mathrm{año}$, y la correspondiente a las hojas de $0.27 \mathrm{~kg} / \mathrm{m}^{2} /$ año, equivalente a 6.6 hojas por año. Estas productividades son bajas en comparación con la media de cultivos anuales (1-2 $\left.\mathrm{kg} / \mathrm{m}^{2} / \mathrm{año}\right)$, pero altas para la productividad típica de las zonas áridas $\left(0.1 \mathrm{~kg} / \mathrm{m}^{2} / \mathrm{año}\right)$. La disponibilidad de agua en el suelo es el factor ambiental con mayor repercusión en la productividad (Nobel y Quero, 1986).

\section{Reproducción}

Agave lechuguilla se puede reproducir sexual y asexualmente (Flores, 1986; Freeman, 1973; Freeman et al., 1977; Eguiarte y Silva, 2000; Freeman y Reid, 1985). Como la mayoría de las especies de Agave, la lechuguilla muere después de florecer y fructificar (Freeman y Reid, 1985; Rzedowski, 1964; Sheldon, 1980). Para llegar a la madurez sexual, la planta requiere desde cuatro hasta 15 o incluso hasta 20 años (Ber- 
langa et al., 1992a; de la Garza, 1987; Freeman y Reid, 1985). Si la planta está sometida a un sistema de aprovechamiento cuidadoso (recolección de los cogollos sin afectar el meristemo apical), la vida de la planta puede prolongarse cinco o seis años más (Sheldon, 1980). La floración de Agave lechuguilla ocurre entre mayo y junio, pero en los años con sequía puede suceder en octubre o noviembre. Cuando las plantas florecen, tienen 51 hojas en promedio (entre 42 y 69 ) y conforman el $6 \%$ de la población en la región de El Paso, Texas. (cuadro 1) (Freeman y Reid, 1985). En promedio, el escapo floral se desarrolla totalmente en 25 días, y su mayor taza de crecimiento la alcanza en los primeros diez días, $20 \mathrm{~cm} /$ día. Las flores están abiertas durante $96 \mathrm{~h}$; las anteras tienen polen disponible $24 \mathrm{~h}$ después de la antesis, y aunque el estigma es receptivo hasta después de las $66 \mathrm{~h}$, la autofertilización sí es posible (Freeman y Reid, 1985). El néctar de las flores está compuesto de 50\% de glucosa, $40 \%$ de fructosa y sólo $10 \%$ de sacarosa; con base en estos datos Freeman y Reid (1983) infieren que los visitadores florales son aves passeriformes, murciélagos, mariposas, algunas abejas, colibríes y entre ellos deben estar los polinizadores. Eguiarte y Silva (2000) agregan a los esfíngidos a esta lista. El volúmen del néctar varía de $50 \mu \mathrm{l}$ en poblaciones del norte de México a $190 \mu \mathrm{l}$ en algunas poblaciones del sur (Eguiarte y Silva, 2000). Al final de la etapa reproductiva, son comunes los individuos con escapos que contienen gran cantidad de semillas viables. Aunque las semillas carecen de letargo, la germinación es óptima con temperatura de 25 a $35^{\circ} \mathrm{C}$; la germinación se reduce drásticamente con menos de 10 y más de $40^{\circ} \mathrm{C}$, temperaturas propias de las épocas fría y cálida, respectivamente, en el hábitat natural de la lechuguilla (Freeman, 1973; Freeman et al., 1977).
Freeman y Reid (1985) observaron que la reproducción de la lechuguilla es casi exclusivamente asexual, por renuevos rizomáticos llamados vástagos, hijatos, retoños o hijuelos. La producción continua de hijuelos, origina grandes y densas agrupaciones de plantas (cuadro 2). Aunque se desconoce la taza de producción natural de retoños, se sabe que la recolección del cogollo y extirpación de hijatos, induce su regeneración y la reducción de su emisión, respectivamente (Berlanga et al., 1992a).

\section{Distribución y hábitat}

Agave lechuguilla es el taxon del subgénero Littae con distribución geográfica más amplia; según Nobel (1998), es una especie que se presenta en $100000 \mathrm{~km}$, desde Texas y Nuevo México, hasta Querétaro, Hidalgo y Guanajuato; además, es probable que existan más individuos de lechuguilla, que de cualquiera de las más de 125 especies de Agave que existen en México (García y Galván, 1995; Gentry, 1982; Marroquín et al., 1981; Nobel, 1998). Según Reyes y Aguirre (1999), la distribución de A. lechuguilla corresponde al patrón altiplanicie mexicana y zonas adyacentes, mientras que para Rzedowski (1978) esta especie es un elemento de las provincias altiplanicie y planicie del noreste, de la región xerofítica mexicana.

Agave lechuguilla es uno de los dominantes fisonómicos del matorral desértico rosetófilo (Rzedowski, 1965) o matorral crasirrosulifolio espinoso (Berlanga et al., 1992a). Para este tipo de vegetación, Miranda y Hernández (1963) reconocieron al subtipo lechuguillal, con base en el nombre que los campesinos asignan a la vegetación donde la lechuguilla domina su fisonomía. La lechuguilla también ha sido registrada en el matorral desértico micrófilo (Rzedowski, 1965), o matorral inerme parvifolio (Berlanga

Cuadro 2. Densidades de Agave lechuguilla en varias localidades de México.

\begin{tabular}{lll}
\hline & Localidad & Individuos/ha (Fuentes) \\
\hline Noria de Gpe., Zac & 56000 & Orozco et al. (1977) \\
Imágenes, Coah. & 41666 & Flores y Perales (1989) \\
Muralla, Coah. & 28333 & Flores y Perales (1989) \\
Saltillo, Coah. & 28221 & Flores y Perales (1989) \\
Parras, Coah. & 26332 & Flores y Perales (1989) \\
La Ventana, Coah. & 26200 & Zapién (1981) \\
Noreste de Zac. & 25128 & Aldrete (1981) \\
Gral. Cepeda, Coah & 24666 & Flores y Perales (1989) \\
Zertuche, Coah. & 23333 & Flores y Perales (1989) \\
Hipólito, Coah. & 22000 & Flores y Perales (1989) \\
Norte de S.L.P. & 21000 & Martínez (1936) \\
La Sauceda, Coah. & 4300 & Berlanga et al. (1992a) \\
\hline
\end{tabular}


et al., 1992a) de Larrea tridentata y Flourensia cernua, lo cual en realidad puede corresponder a extensos ecotonos entre el matorral rosetófilo y el micrófilo, como fue registrado por Aldrete (1981).

En el matorral desértico rosetófilo $A$. lechuguilla alcanza las mayores dominancias fisonómicas y cuantitativas. Así, en el noreste de Zacatecas, donde es una de las especies más importantes por sus altas densidades (cuadro 2); A. lechuguilla obtuvo en promedio un valor de importancia absoluto de 30.13 cuando para todo el estrato arbustivo dicho valor fue de 87.51 (Aldrete, 1981). El matorral desértico rosetófilo ocurre en sierras y cerros de origen sedimentario o con mezcla de este sustrato, y desciende hasta las partes media y baja de sus laderas, generalmente entre los 1000 y los 2700 msnm (Rzedowski, 1965), pero A. lechuguilla suele encontrarse entre los 950 y los $2300 \mathrm{msnm}$ (Marroquín et al., 1981). Sin embargo, Berlanga et al. (1992a), de la Garza (1987) y Gentry (1982) afirman que esta especie se distribuye desde los 200 hasta los 2400 msnm. Dada la amplitud altitudinal, el matorral desértico rosetófilo de lechuguilla se localiza en todos los tipos climáticos del área: secos (BW), semisecos $\left(\mathrm{BS}_{0}\right.$ y $\left.\mathrm{BS}_{1}\right)$ y templados subhúmedos $\left(\mathrm{Cw}_{0}\right)$, con temperaturas extremas de -8 a $44^{\circ} \mathrm{C}$, y precipitación entre 150 y $500 \mathrm{~mm}$ anuales (Berlanga et al., 1992a; Gentry, 1982). En los lechuguillales del noreste de Zacatecas estudiados por Aldrete y Aguirre (1982), el sustrato geológico es de rocas sedimentarias (caliza, conglomerado, lutita y arenisca), pero a veces se localizan también sobre rocas ígneas; los suelos ge- neralmente son litosoles con profundidades menores de $10 \mathrm{~cm}$; aunque hay sitios con suelos de $45 \mathrm{~cm}$, estos corresponden a xerosoles, regosoles, fluvisoles o feozems. Según los autores citados, dichos suelos presentaron, en general, $37.3 \%$ de arena, $34.5 \%$ de arcilla, $28.3 \%$ de limo, $4 \%$ de materia orgánica, $3 \%$ de carbono orgánico, 1.2 ppm de fósforo extraíble y $58.6 \%$ de carbonatos, conductividad eléctrica menor de 2 mmhos/cm y pH de 7.7. En cuanto a la condición de su superficie, en estos suelos se registró en promedio $15.2 \%$ de área desnuda, $34.3 \%$ ocupada por piedras, $27.7 \%$ con mantillo, $13 \%$ con vegetación y $0.4 \%$ con heces (Aldrete y Aguirre, 1982).

Altitudinalmente, el matorral desértico rosetófilo se encuentra entre 1500 y $2700 \mathrm{msnm}$; por la parte más alta colinda con el piñonar, el encinar o el pastizal, y por la parte más baja con el matorral desértico micrófilo o el matorral crasicaule (Aldrete, 1981). Por otro lado, el matorral desértico rosetófilo se caracteriza por su estratificación compleja (cuadro 3) y gran riqueza florística. Al respecto, Reyes et al. (1996) registraron 122 especies en este matorral, riqueza muy superior a la del matorral desértico micrófilo (53 especies) y casi tan alta como la del matorral crasicaule (145 especies), presentes en una sierra, $20 \mathrm{~km}$ norte de Charcas, S.L.P.

La densidad de A. lechuguilla es notablemente variable. Se han documentado de 4300 a 56000 individuos/ha; sin embargo, las estimaciones más frecuentes oscilan entre 21000 y 28333 individuos/ha (cuadro 2). Por su parte, Zapién (1981) estimó 8100 hijuelos/

Cuadro 3. Estratos y dominantes fisonómicas en el matorral desértico rosetófilo del noreste de Zacatecas.

Elementos subarbóreos aislados (2.0-3.5 $\mathrm{m}$ de altura):

Estrato arbustivo superior (2.0-2.5 $\mathrm{m}$ de altura):

Estrato arbustivo medio (0.7-1.2 $\mathrm{m}$ de altura):

Estrato arbustivo inferior (de 0.3-0.5 $\mathrm{m}$ de altura):

Estrato herbáceo (de 0.03-0.2 $\mathrm{m}$ de altura):
Yucca carnerosana (palma samandoca) y Fouquieria splendens (ocotillo)

Acacia constricta (huizache), Larrea tridentata (gobernadora), Sophora secundiflora (colorín), Mortonia greggi, Lindleyella mespiloides y Rhus virens.

Ephedra aspera (popotillo), Dasylirion leiophyllum (sotol), Parthenium argentatum (guayule) y Chrysactinia mexicana (hierba de San Nicolás).

Agave lechuguilla (lechuguilla), Euphorbia antisiphylitica (candelilla), Opuntia rastrera (nopal rastrero), Hechtia glomerata (guapilla china) y Agave striata (espadín).

Dyssodia pentachaeta, D. acerosa, D. setifolia, Nama palmeri, Loeselia scariosa, Bouteloua curtipendula, Leptochloa dubia, Panicum halli y Aristida divaricata. 
ha y entre 18100 a 23200 plantas aprovechables por hectárea en La Ventana, Coah., una región donde se aprovecha intensamente la lechuguilla.

\section{Composición química}

Orozco et al. (1977) estimaron que el peso fresco promedio de un cogollo es de $356.54 \mathrm{~g}$ y contiene $14.6 \%$ de fibra y $85.43 \%$ guishe (tejido suculento y agua de las hojas). La palabra guiche, guishe, guishi, guixi o guizo se ha atribuido de origen otomí y se aplica "...a la irritación o escozor que deja el jugo crudo del maguey en alguna parte del cuerpo..." (Santamaría, 1992). Sin embargo el término náhuatl mexixi (de metl, maguey y posiblemente xixicoa, hacer daño o pena) aplicado a la epidermis carnosa y gruesa de las pencas del maguey (Simeón, 1977), podría aludir a los jugos cáusticos y ser el verdadero origen de guishe. Nobel (1988) registró en el clorénquima: $1.14 \%$ de N, 1220 ppm de P, $1.27 \%$ de K, 45 ppm de $\mathrm{Na}, 6.11 \%$ de $\mathrm{Ca}, 0.40 \%$ de $\mathrm{Mg}, 14 \mathrm{ppm}$ de $\mathrm{Mn}, 7 \mathrm{ppm}$ de $\mathrm{Cu}, 36 \mathrm{ppm}$ de $\mathrm{Zn}$ y $77 \mathrm{ppm}$ de Fe.

Algunos autores aseguran que la presencia de la saponina hepatonefrotoxina (glucósido derivado de un triterpeno tetracíclico, de 27 átomos de carbono), termoestable e hidrosoluble, hace tóxica la lechuguilla para el ganado (Aguilar y Zolla, 1982; Gentry, 1982). Además, el contacto del guishe con la piel humana produce dermatitis y puede o no estar acompanada por fotosensibilidad de la parte afectada. Aunque se desconoce la causa de este efecto, se ha sugerido que el factor responsable podría ser un pigmento fotodinámico contenido en las plantas (Aguilar y Zolla, 1982). Otra posibilidad es que las sapogeninas contenidas en el guishe, por sus características anfipáticas (su estructura contiene una fracción hidrosoluble polar, y otra hidrofílica no polar), o de detergente, alteren las membranas celulares, por lo que pueden causar dolor e incluso la muerte de animales (Nobel, 1998).

\section{Interacciones negativas de $A$. lechuguilla con animales}

Flores y Perales (1989) realizaron muestreos sistemáticos en siete localidades ixtleras del sur de Coahuila, para registrar los enemigos naturales de $A$. lechuguilla. Para ello, en cada localidad marcaron una área de observación y recolecta, y la visitaron periódicamente durante un año. Los insectos más perjudiciales para la planta fueron los picudos (Peltophorus polymitus y Scyphophorus interstitialis) y el gusano barrenador (Megathymus sp.) (cuadro 4).

En el caso de $P$. polymitus, coleóptero curculiónido, las hembras perforan la superficie del escapo para ovipositar; luego, las larvas también perforan el escapo, hasta llegar a su parte central, donde preparan cámaras pupales. Las mayores cantidades de larvas se alcanzan entre julio y agosto y pueden registrarse entre 70 y 120 larvas por escapo. De la pupa emerge un adulto, el cual en agosto perfora un orificio nuevo para salir. El adulto se alimenta de hojas maduras e inmaduras y del escapo; en las hojas realiza perforaciones concéntricas que pueden variar desde 10 hasta 100 por hoja, con un diámetro de $1.0 \mathrm{~cm}$ (Flores y Perales, 1989).

Las hembras del curculiónido S. interstitialis ovipositan entre marzo y mayo en los tejidos en descomposición o en los tejidos suaves de las hojas maduras. Cuando las larvas emergen del huevo, perforan la hoja hasta la raíz, en donde completan el estadio pupal; entre agosto y septiembre emergen los adultos y mediante una perforación salen del interior de la planta. Los adultos pasan el día refugiados en la axila de la hojas, en donde hacen perforaciones subepidérmicas para obtener alimento, aunque también pueden barrenar hasta la raíz para consumirla (Flores y Perales, 1989).

Por otro lado, la hembra de Megathymus sp., una mariposa de la familia megatímide, en marzo oviposita en el ápice del cogollo y cubre los huevecillos con una sustancia gomosa y cristalina. Después de 15 días, las larvas perforan el ápice del cogollo y para salir descienden un poco por la superficie del cogollo y se introducen nuevamente en él a través de una nueva perforación. Después, un poco más abajo, hacen una perforación de salida y repiten el proceso de entrada y salida sucesivamente a todo lo largo del cogollo. Finalmente, las larvas descienden al interior de la planta, hasta la parte inferior del tallo, en donde hacen una cámara pupal, cerrada con una capa cerosa, y después de 30 días, a principios de septiembre, emerge el adulto. De esta forma, el daño a la planta es total (Flores y Perales, 1989).

Otras plagas de la lechuguilla son las escamas, homópteros que cubren la superficie de las hojas; los grillos y chapulines, ortópteros que sólo depredan el ápice de las hojas y pueden provocar la muerte de la hoja cuando el consumo es intenso. Los topos consumen las raíces; $y$ roedores y lagomorfos mordisquean la base de las hojas (Flores y Perales, 1989).

Sobre el uso forrajero de la lechuguilla existen discrepancias. Algunos autores (Escobar, s. f.; Magallán, 1998; Zapién, 1981) aseguran que la lechuguilla es un forraje excelente, que se puede usar en casos de sequías extraordinarias, pero probablemente sólo se refieren al escapo floral (A. Silva, com. pers.), pues otros autores (Aguilar y Zolla, 1982; Gentry, 1982; Gómez, 1965) afirman que la lechuguilla es tóxica para 
el ganado. En efecto, se ha documentado que vacas, cabras y borregos han muerto después de consumir las hojas de la planta. Además, se ha señalado que el principio tóxico es la saponina hepatonefrotoxina (Aguilar y Zolla, 1982; Gentry, 1982). Los síntomas iniciales en los animales que han consumido la planta son semejantes a los de la hepatitis y nefritis: pérdida de apetito, indiferencia, debilidad progresiva, edemas en la cabeza y orejas y una secreción amarillenta de los ojos y las pezuñas; estas alteraciones conducen al animal al estado de coma y luego a la muerte. En la necropsia, los tejidos muestran color amarillo, hepatomegalia y lesiones ictéricas en hígado y riñones (Aguilar y Zolla, 1982).

\section{Usos}

Existe evidencia arqueológica del uso de la fibra de lechuguilla por los habitantes prehispánicos de las zonas áridas del norte de México; en Cuatro Ciénegas, Coah, se registraron sandalias hechas con dicha fibra, de aproximadamente 8000 años de edad (Crane y Griffin, 1958; Rodríguez, 1985). Los chichimecas en general, fueron hábiles para la manufactura de cuerdas y cestos con fibras de plantas de la región (entre las que se incluyó la lechuguilla); también fabricaron sandalias, redes y cobertores mortuorios (Rodríguez, 1985; Valdés, 1995); en la región de Tierra Nueva, S.L.P., se encontraron cestas para transportar a los lactantes, elaboradas por los Guachichiles (Rodríguez, 1985). Los Irritilas, chichimecas de la región actual de La Laguna (estados de Coahuila y Durango), pescaban con unas redes cónicas que estaban hechas con fibras de izote (Yucca spp.) y de lechuguilla; esas redes todavía se usan y se conocen como nazas, de donde procede el nombre actual del río (Valdés, 1995). León (1649, citado por Sheldon, 1980) afirma que los chichimecas sometían a cocción los co- gollos de la lechuguilla, les denominaban mezcal, y los consumían masticándolos. Según Nobel (1998), los indígenas obtenían un extracto de la lechuguilla con el que envenenaban las puntas de las flechas; al menos hasta 1957, los tarahumaras preparaban una maceración de hojas de lechuguilla que luego vertían en los ríos, para propiciar la muerte de peces (Pennington, 1957).

En México, el aprovechamiento comercial de la lechuguilla se inició en las haciendas, durante la segunda mitad del siglo XIX. Entre 1890 y 1896 se exportaban cerca de $6 \mathrm{t}$ anuales de ixtle (fibra dura de la lechuguilla) (Sheldon, 1980). Actualmente, el maguey espontáneo más abundantemente recolectado para la producción de fibra es la lechuguilla y más de 200000 recolectores la procesan anualmente (Nobel, 1998). La producción anual en los últimos años ha variado entre 3400 y $5200 \mathrm{t}$ (J.A. Valdez, com. pers.). El ixtle de lechuguilla recibe varios nombres (ixtle, istle, Mexican fibre o Mexican fiber, Tula fiber, Tampico fibre o Tampico fiber y Tampico hemp); el nombre de fibra de Tampico, proviene del nombre del puerto mexicano por donde se exporta (Dewey, 1965; Gentry, 1982; Kirby, 1950). La mayor parte de la fibra producida se exporta a países productores de cepillos industriales, como Estados Unidos, Suiza, Japón, Holanda, Alemania y Gran Bretaña (García y Sicilia, 1984; Kirby, 1950); pero también se exporta fibra de lechuguilla a Argentina, Brasil, Canadá, España, Francia, Italia y Venezuela (Nieto, 1983). Las virtudes de esta fibra son su suavidad y resistencia; se le usa en la industria automovilística, para elaborar tapetes y bajo-alfombras, en cordelería y cepillería, para la fabricación de bolsas, sacos y mantas, para cepillos de los aparatos de limpieza y pulido de alfombras y pisos, en la fabricación de varios tipos de brochas, en la limpieza y pulido de maquinaria de acero, y los sobrantes se usan para hacer estropajos y relle-

Cuadro 4. Plantas dañadas y hojas afectadas por animales en poblaciones de A. lechuguilla al sur de Coahuila.

\begin{tabular}{|c|c|c|c|c|c|c|}
\hline & \multicolumn{6}{|c|}{ Animales } \\
\hline & $\begin{array}{c}\text { Peltophorus } \\
\text { polymitus } \\
\text { (picudo) }\end{array}$ & $\begin{array}{c}\text { Scyphophorus } \\
\text { interstitialis } \\
\text { (picudo) }\end{array}$ & $\begin{array}{l}\text { Homópteros } \\
\text { (Escamas) }\end{array}$ & $\begin{array}{l}\text { Ortópteros } \\
\text { (grillos y } \\
\text { chapulines) }\end{array}$ & $\begin{array}{c}\text { Megathymus } \\
\text { sp. (gusano } \\
\text { barrenador) }\end{array}$ & $\begin{array}{c}\text { Roedores } \\
\text { y lagomorfos }\end{array}$ \\
\hline Plantas dañadas (\%) & 77.4 & 74.2 & 50.6 & 23.5 & 14.1 & 10.2 \\
\hline $\begin{array}{l}\text { Hojas afectadas } \\
\text { por individuo (\%) }\end{array}$ & 32.8 & 24.7 & 16.8 & 11.7 & 100 & 10.4 \\
\hline
\end{tabular}

(Fuente: Flores y Perales, 1989) 
no de muebles (Berlanga et al., 1992a; Flores y Perales, 1989; Kirby, 1950; Rzedowski, 1964; Sheldon, 1980). El ixtle reforzado con resinas termoplásticas o termofijas se usa para fabricar láminas semejantes a las de fibra de vidrio, que a su vez, se utilizan en la manufactura de tinacos, muebles, accesorios para el hogar y lanchas deportivas (Belmares et al., 1979b; 1985; Nieto, 1983). Existe la posibilidad de extraer celulosa de la fibra de lechuguilla, pues se obtienen rendimientos de 40 a $70 \%$ de celulosa y $18 \%$ de lignina y hemicelulosa (Belmares et al., 1979a; 1979b; Márquez et al., 1996); aunque la longitud y anchura de las células (0.92 mm y 27 micras) no son las más indicadas para elaborar papel, que requiere material vegetal con células más largas y angostas (McLaughlin y Schuck, 1991). Las características físicas de la fibra de lechuguilla son una resistencia a la tensión entre 172 y 241 kilonewtons por kilogramo, una capacidad de alargamiento entre 12 y $23 \%$, y una densidad de 1.3 a $1.5 \mathrm{~g} / \mathrm{cm}^{3}$ (Belmares et al., 1979a; 1979b).

El guishe tiene características físicas y químicas favorables, como su poder abrasivo, pero hasta ahora ha sido poco aprovechado, pues podría tener otros usos como la elaboración de láminas, maderas aglomeradas, cartón, papel filtro y esteroides (Berlanga et al., 1992a). Del tallo y del guishe se obtienen saponinas para fabricar en forma industrial jabones y champús (Flores y Perales, 1989; Zapién, 1981); también se extrae una sustancia activa contra el agente causal de la tuberculosis, el basilo Mycobacterium tuberculosis; dicha sustancia se inyecta al ganado ovino y vacuno para su protección (Flores y Perales, 1989).

Respecto a su uso como fuente de esteroides, $A$. lechuguilla es materia prima para la obtención de esmilagenina, que es un precursor esteroidal, ya que sus hojas contienen entre 1 y $2 \%$ del peso seco, de esa sapogenina (Wall et al., 1962). También, existe la posibilidad de obtener de la lechuguilla la materia prima para la elaboración de cortisona, un antiinflamatorio, e incluso estrógeno y progesterona, hormonas sexuales femeninas (Nobel, 1998). Se ha documentado la actividad insecticida de los extractos de guishe (Castro-Franco et al., 1998), por lo que esta aplicación podría sumarse a la lista de usos ya mencionada.

Los ixtleros o talladores (recolectores de $A$. lechuguilla), además de preparar la fibra de la lechuguilla para su venta, también aprovechan otras partes de esta planta. El uso doméstico más común de la fibra es para la elaboración de cordeles, cuerdas o mecates que se usan para arrastrar, amarrar o cargar objetos o animales; se usan para tender al sol la ropa mojada, para cinchos de monturas, para amarrar los ar- neses de la yunta, para hacer bridas, estropajos, escobetas y otros objetos. La venta de cuerdas, escobetas o estropajos, en las ciudades cercanas a donde se produce la fibra de lechuguilla, es un ingreso económico importante para los recolectores (Sheldon, 1980)

En Mapimí, Durango se consumen las flores de lechuguilla como verdura (Montúfar, 1987). Escobar (s.f.) consignó el uso de la lechuguilla para, al igual que con Dasylirion sp., fabricar sotol, una bebida alcohólica destilada.

Los ixtleros usan el tallo y las raíces de la lechuguilla como amole (del náhuatl amolli, jabón; Santamaría, 1992). Al agitar un pedazo de tallo en el agua, produce una solución jabonosa. Esta solución se puede usar como champú, jabón para el cuerpo y para la ropa; fue el amole preferido por los tarahumaras para lavar ropa blanca (Pennington, 1957; Sheldon, 1980). Martínez (1936) observó en los mercados la venta de raíces de lechuguilla para lavar la ropa, pero especialmente para lavar la lana. La propiedad jabonosa también la tienen las hojas de la lechuguilla (Sheldon, 1980), las cuales se cortan en fragmentos y reciben el nombre de shishi (Martínez, 1936). La combinación de la saponina de las hojas con las espinas marginales, hacen del guishe con bagazo un material abrasivo ideal para eliminar el hollín de ollas y cazuelas. Los escapos florales se suelen usar como material para construcción de paredes o techos de las chozas, y como combustible (Juárez et al., 1996; Sheldon, 1980).

\section{Recolección}

Desde hace varias décadas, la oferta de fibras duras de las zonas áridas y semiáridas del norte de México es mantenida por los campesinos recolectores, llamados ixtleros, puyeros o talladores, quienes viven en una parte del área de la distribución de la lechuguilla (estados de San Luis Potosí, Zacatecas, Coahuila, Nuevo león y Tamaulipas) (Gentry, 1982; Sheldon, 1980). Preferentemente se recolecta el cogollo, aunque también se pueden usar las hojas desplegadas (Nieto, 1983; Berlanga et al., 1992a). Los cogollos pueden recolectarse cuando la planta tiene alrededor de seis años de edad (Sheldon, 1980), o cuando el cogollo tiene de 42 a $60 \mathrm{~cm}$ (Nobel, 1998; Zapién, 1981). Los cogollos se desprenden por torsión, con una navaja, o con una cogollera; ésta es una pértiga de 1-1.5 m de largo con una argolla metálica en un extremo, la cual se introduce por el cogollo y con un ligero movimiento de palanca se le desprende de la planta dejando un cogollito donde prosigue un nuevo crecimiento (de la Garza, 1987; Dewey, 1965; 
Martínez, 1936). Los cogollos se acumulan en cestas llamadas huajacas (Dewey, 1965; de la Garza, 1987). Para obtener $6 \mathrm{~kg} /$ fibra/día, que es la máxima producción posible registrada por hombre, se deben recolectar unos 193 cogollos, que en conjunto pesan aproximadamente $69 \mathrm{~kg}$ (Orozco et al., 1977).

Cuando el cogollo se recolecta cuidadosamente, la planta lo regenera. El tiempo necesario para la regeneración varía y depende de la precipitación; si la lluvia es abundante, la regeneración tarda seis meses y si es escasa, toma un año (González y Scheffey, 1964). La velocidad de recuperación del cogollo es de 1.0 a $2.5 \mathrm{~cm} / \mathrm{mes}$ (Zapién, 1981; Berlanga et al., 1992a, 1992b), pero se desconoce con certeza su relación con la cantidad de lluvia. En años en los que llueve suficiente y a tiempo, la lechuguilla puede generar altos rendimientos de fibra, pero el campesino prefiere dedicarse a la producción de cosechas. En cambio, cuando hay sequía, el crecimiento de la planta es lento, y es cuando la lechuguilla se aprovecha principalmente (Rzedowski, 1964).

La fibra se obtiene por descortezamiento o tallado (Belmares et al., 1985); el objetivo es eliminar la epidermis de la hoja y separar las fibras del guishe o bagazo (tejido suculento de la hoja); para cumplir el objetivo, existen el método manual y el mecánico. En el proceso manual se tallan principalmente las hojas tiernas del cogollo (Dewey, 1965); aunque este proceso es más lento y sus rendimientos son menores, la fibra que se obtiene es de mayor calidad (fibras largas, uniformes y con mayor resistencia). En la extracción mecánica, las hojas del cogollo y del resto de la roseta se tallan con máquinas (Dewey, 1965), y aunque el proceso es más rápido y supuestamente más eficiente, se obtiene fibra de menor calidad (Berlanga et al., 1992a). Independientemente del método empleado, usualmente se utilizan sólo las hojas o pencas más grandes, para lo cual el cogollo recolectado se abre y se eliminan las hojas más pequeñas del centro (Berlanga et al., 1992a; Rzedowski, 1964).

La talla manual se realiza en el lugar de la recolecta, cuando el lechuguillal está alejado de la casa del recolector; o en el solar de la casa, si el transporte desde el área de recolección no implica problemas (de la Garza, 1987). Para la talla, se improvisa o se busca una sombra. Primero, a las hojas se les eliminan las espinas marginales y el ápice (Dewey, 1965; Nobel, 1998); para despulparlas se usa un instrumento llamado tallador, que es un cuchillo largo con filo romo, con el cual se presiona la hoja contra un pedazo de madera que se llama banco. El tallador tiene la punta en forma de gancho para insertarlo en un extremo del banco. Al momento de presionar la hoja, el recolector la jala hacia él; primero talla de la parte media de la hoja hacia el ápice, lo que se llama despunte, y luego de la parte media a la base de la hoja, lo que se llama destronque. El proceso se repite cuantas veces sea necesario, por lo general cinco veces por hoja, hasta eliminar todo el tejido suculento y dejar sólo la fibra (Dewey, 1965). En este proceso también se puede usar un trozo de madera llamado bolillo sobre el que enredan los extremos de las hojas para facilitar el tirar de ellas; la parte gruesa del bolillo se usa durante el despunte y la delgada para el destronque. Finalmente, la fibra se extiende para secarse al sol, durante unos dos o tres días (Berlanga et al., 1992a; Gentry, 1982; Martínez, 1936; Rzedowski, 1964; Zapién, 1981). El color de la fibra es blanco y opaco, con un ligero tinte verdoso, el cual es más pronunciado cuanto más imperfecto haya sido el proceso de la talla. La longitud de la fibra está determinada por la longitud de la hoja (Belmares et al., 1985), la cual puede medir entre 42 y $60 \mathrm{~cm}$ (Zapién, 1981; Nobel, 1998). La fibra representa del 6.2 al 9.12\% (Berlanga et al., 1992a; Zapién, 1981) del peso de la hoja, proporción superior a la del henequén y el sisal, que es de 3 a $6 \%$ (Belmares et al., 1985).

Otros dos métodos para obtener la fibra son la extracción total de la planta, para luego desprender todas las hojas y desechar el tallo y cogollo. Para esto el tallador usa una tenaza de resorte colocada en un bloque de madera y empieza el tallado, deshechando la base de las hojas. En el tercer método la fibra se obtiene sin desprender la planta del suelo, con una tenaza filosa, que se coloca en la base de la hoja y se jala fuertemente para eliminar el parénquima; después de hacerlo con todas las hojas de la planta, la fibra se desprende con un cuchillo (Marroquín et al. 1981).

Para la talla mecánica se han comercializado varias máquinas desfibradoras, como la Beard Hyne, Prieto, Clamon, Farías, Winfield, Jhones y Pons (Escobar, s. f.), que por ser más rápidas, se han considerado más eficientes que los talladores humanos; sin embargo, ninguna supera la calidad de la fibra obtenida manualmente, ya que las máquinas generan fibras de tamaño heterogéneo y baja resistencia (Berlanga et al., 1992a; Dewey, 1965; Zapién, 1981). La máquina más popular y rústica consiste en un cilindro de mezquite de $20 \mathrm{~cm}$ de diámetro por $20 \mathrm{~cm}$ de longitud, con hileras de clavos romos, separadas cada $3 \mathrm{~cm}$. El cilindro está colocado sobre una armazón metálica y cubierto por una caja de madera, con una abertura horizontal en la parte frontal. Por la abertura se introduce manualmente la mitad superior de una hoja, luego sin soltarla se recupera y se introduce la otra mitad de la hoja. El cilindro se mueve con un motor eléctrico o de gasolina, colocado en 
la parte inferior de la armazón de metal (Berlanga et al., 1992b; de la Garza, 1987). En general, las máquinas probadas oprimen demasiado la fibra, la fraccionan, le restan elasticidad y la dejan muy sucia (de la Garza, 1987; Dewey, 1965); además, con cierta frecuencia las máquinas rústicas han producido mutilaciones en las manos de los operarios. Las máquinas despulpadoras usadas para el henequén o el sisal son inadecuadas para las hojas de lechuguilla, por su corta longitud (Dewey, 1965); sin embargo, podrían tomarse como base para diseñar una máquina moderna para lechuguilla y para las condiciones de los lechuguilleros.

Una investigación en 135 comunidades lechuguilleras de cinco estados de la república (cuadro 5) demostró que, en promedio, $17 \%$ de los habitantes de las comunidades evaluadas se dedicaba a la recolección de lechuguilla (de la Garza, 1987). Sin embargo, la cantidad de ixtleros o talladores en una comunidad está relacionada positivamente con la sequía, ya que si la precipitación es favorable, la po- blación se dedica a la producción de cosechas (Sheldon, 1980). La distancia media de los poblados a las áreas de recolección es de $5.94 \mathrm{~km}$. En general, se talla de cinco a seis días a la semana, y sólo durante una tercera parte del año; esa parte del año corresponde a los meses más secos (de la Garza, 1987). La eficiencia en la obtención manual de la fibra depende de diversos factores, como la calidad de los cogollos, distancia a los sitios de recolección y habilidad o destreza del recolector. El rendimiento individual es desde a $4.5 \mathrm{~kg} /$ fibra/día (Flores y Perales, 1989) hasta $6 \mathrm{~kg} /$ fibra/día (Orozco et al., 1977). Aunque podrían esperarse incrementos en el rendimiento con el uso de desfibradoras, de la Garza (1987) encontró que con la adquisición de desfibradoras en los ejidos, la producción de ixtle por persona se mantiene igual, pues los campesinos prefieren usar el tiempo extra para realizar otras actividades o descansar, debido al precio bajo de la fibra. Es de notar que en la estimación de la eficiencia de las máquinas, general-

Cuadro 5. Recolección de Agave lechuguilla en 135 comunidades de cinco estados de México.

\begin{tabular}{|c|c|c|c|c|c|}
\hline & S.L.P. & Coah. & N.L. & Zac. & Tamps. \\
\hline & \multicolumn{5}{|c|}{ Municipios (número de comunidades analizadas) } \\
\hline & $\begin{array}{l}\text { Guadalcázar } \\
\text { (9), Matehuala } \\
\text { (5) Cedral (7) }\end{array}$ & $\begin{array}{l}\text { Parras (13) } \\
\text { Gral. Cepeda (10) } \\
\text { Ramos Arizpe (12) }\end{array}$ & $\begin{array}{c}\text { Aramberri (7) } \\
\text { Dr. Arroyo (29) } \\
\text { Galeana (5) }\end{array}$ & $\begin{array}{l}\text { Mazapil (8) } \\
\text { Concepción } \\
\text { del Oro (6) }\end{array}$ & $\begin{array}{c}\text { Jaumave (8) } \\
\text { Bustamante (8) } \\
\text { Tula (8) }\end{array}$ \\
\hline Ixtleros por comunidad (\%) & 21.74 & 12.23 & 14.59 & 18.19 & 20.52 \\
\hline Distancia a los lechuguillales ( $\mathrm{km})$ & 5.68 & 8.75 & 4.23 & 5.45 & 5.44 \\
\hline Días/mes dedicados a la talla & 15.87 & 19.14 & 22.51 & 21.98 & 21.52 \\
\hline Días/año dedicados a la talla & 117.08 & 170.76 & 166.11 & 166.71 & 172.15 \\
\hline $\begin{array}{l}\text { Ixtle/día /persona obtenido } \\
\text { manualmente }(\mathrm{kg})\end{array}$ & 3.94 & 5.01 & 4.60 & 4.99 & 3.15 \\
\hline $\begin{array}{l}\text { Ixtle /día/persona obtenido } \\
\text { mecánicamente }(\mathrm{kg})\end{array}$ & 15.00 & 14.59 & 11.38 & 0.00 & 0.00 \\
\hline $\begin{array}{l}\text { Ixtle /mes/persona obtenido } \\
\text { manualmente }(\mathrm{kg})\end{array}$ & 61.33 & 101.04 & 90.53 & 112.09 & 67.32 \\
\hline $\begin{array}{l}\text { Ixtle /mes/persona obtenido } \\
\text { mecánicamente }(\mathrm{kg})\end{array}$ & 300.00 & 261.71 & 148.0 & 0.00 & 0.00 \\
\hline $\begin{array}{l}\text { Ejidos con máquinas } \\
\text { desfibradọras }(\%)\end{array}$ & 14.29 & 61.79 & 9.76 & 0.00 & 0.00 \\
\hline
\end{tabular}

(Fuente: de la Garza, 1987) 
mente no se considera el tiempo requerido para la recolección de los cogollos, y que las máquinas pueden funcionar continuamente.

Berlanga et al. (1992a), con base en los cálculos de Morales (1988) diseñaron un método de campo para estimar la existencia de fibra en lechuguillales. El método consiste en muestrear una población por el método de parcelas (los autores sugieren de $100 \mathrm{~m}^{2}$ ), o de cuadrantes centrados en un punto. En cualquiera de los casos, de las plantas incluidas en la parcela o las más cercanas a los puntos de la línea, se registra la altura y el diámetro basal del cogollo, con lo cual se predice su peso en gramos; para ello presentan una tabla con los pesos predichos mediante una ecuación de regresión múltiple, para valores crecientes de altura y diámetro basal de cogollos. Luego se obtiene el peso promedio por cogollo, el cual se multiplica por el total de individuos aprovechables en todo el terreno. La cifra que se obtiene se divide entre 16.13, que es el equivalente al $6.2 \%$ de rendimiento de fibra con tallado manual, o entre 12.5 , equivalente al $8 \%$ de rendimiento con máquina desfibradora. A la fecha, no se han encontrado evidencias sólidas de la relación que pudiera existir entre la cantidad y época de corte de cogollos y la recuperación de la planta o la cantidad y calidad de la fibra (Berlanga et al., 1992a).

\section{Proyectos de conservación}

Desde 1964 se señaló la necesidad de realizar experimentos con A. lechuguilla, para identificar sus condiciones óptimas de reproducción, crecimiento y producción de fibra (Rzedowski, 1964). Berlanga et al. (1992b) desarrollaron un método para la repoblación artificial. Este consiste en recolectar cuidadosamente, sin dañar raíces ni tallo, súrculos o hijuelos de $30 \mathrm{~cm}$ de altura, sanos, libres de plagas, bien conformados, y con cogollo recto y dejarlos al aire libre por 24-48 hrs., para facilitar la cicatrización de las posibles heridas hechas durante la recolección; la aplicación de sustancias químicas para evitar el ataque de microorganismos se considera innecesaria. Luego, la plantación debe realizarse 20 días antes del inicio de la lluvias, en cepas de $10-15 \mathrm{~cm}$ de profundidad, en la parte superior de bordos de $30 \mathrm{~cm}$ de altura, y se les cubre con suelo hasta unos centímetros arriba de la parte basal de las plantas; la densidad debe ser de 20000 plantas/ha, y se recomienda plantar en parcelas abandonadas, en sitios de ladera o planos, con condiciones similares al hábitat de las poblaciones espontáneas. En caso de no contar con parcelas abandonadas, se pueden hacer aclareos en la vegetación y en ellos plantar la lechuguilla. Las plantaciones así generadas, requieren pocos cuidados, entre los que destacan el reemplazo de las plantas muertas, enfermas o con plagas, la reconstrucción de los bordos después de la época de lluvias y el control de insectos nocivos; también recomiendan la remoción de los hijuelos.

Por su parte, Orozco et al. (1977) sugirieron el manejo de las poblaciones naturales de lechuguilla, para lo cual proponen la conservación en el terreno de las especies leñosas grandes, y la eliminación de herbáceas anuales y perennes, con el objeto de abrir espacios para plantar individuos de lechuguilla.

\section{Discusión}

Las plantas del género Agave se distinguen por poseer características extraordinarias que el hombre ha aprovechado a través de miles de años de relación (Gentry, 1982). Con base en la literatura consultada, se puede confirmar que $A$. lechuguilla es una especie sobresaliente, pues es una planta monocárpica polianual (con 15 a 20 años de vida, prorrogables a cinco o más años si el cogollo se cosecha con cuidado), con alta capacidad de competencia intraespecífica (alcanza densidades de 21000 a 28000 individuos/ha) e interespecífica (predomina frecuentemente en el matorral desértico rosetófilo, comunidad de alta riqueza florística), con amplia resistencia al ataque de plagas y a la herbivoría por mamíferos y a condiciones ambientales extremas (temperaturas entre -8 y $44^{\circ} \mathrm{C}$ y precipitaciones anuales entre 150 y $500 \mathrm{~mm}$ ), con productividad biológica ( $0.38 \mathrm{~kg}$ de biomasa seca/ $\mathrm{m}^{2} / \mathrm{año}$ ) superior a la típica de las zonas áridas, distribución geográfica amplia (en $100000 \mathrm{~km}^{2}$ ), capacidad de reproducción vegetativa extraordinaria (cerca de 8100 hijuelos/ha) y capacidad de reproducción sexual elevada (gran producción de semilla sin latencia). Sin embargo, la presión de recolección es tan intensa y el aprovechamiento de los escapos florales para forraje tan alto, que esas altas densidades naturales pueden reducirse hasta 4000 individuos/ha. Esto está obligando a recolectar cogollos en lechuguillales lejanos a los caseríos (de 4 a $8 \mathrm{~km}$ ) y propiciando la recolección de cogollos menores de $40 \mathrm{~cm}$, con lo cual se provoca la muerte inmediata de la planta, al destruirse el meristemo apical.

El problema del deterioro de los lechuguillales no se resuelve con plantaciones en campos agrícolas abandonados (como el propuesto por Berlanga et al., 1992b), pues estos representan una superficie mínima e insuficiente. Así, la solución radica en el aprovechamiento persistente o racional de los lechuguillales espontáneos, como lo propuesto por Orozco et al. (1977), pues sólo así se podrán formular y probar 
planes de manejo de la lechuguilla con posibilidades de ser adoptados por los campesinos ixtleros. Pero para ello, existen varios aspectos aún por investigarse, como los señalados por varios autores (Zapién, 1981; Berlanga et al., 1992a, 1992b): a] el análisis demográfico de las poblaciones de lechuguilla, con especial énfasis en la taza de producción de hijuelos; b] la variación en la producción y calidad de la fibra en relación con la calidad del sitio; $c$ ] la búsqueda, identificación y caracterización de las posibles variantes de lechuguilla y sus calidades de fibra; $d]$ las relaciones que pudieran existir entre la cantidad y época de la recolecta de cogollos, la recuperación de la planta y la cantidad y calidad de la fibra, y el papel que en todo lo anterior juega la precipitación; $e$ ] los efectos del pastoreo y aprovechamiento diverso, como la recolecta de fibra de palma samandoca (Yucca carnerosana), candelilla, sotol (Dasylirion spp.), leña y cacería que se realizan en las comunidades de las que forma parte la lechuguilla. Complementariamente, se requieren estudios etnobotánicos para integrar y analizar el conocimiento tradicional sobre la lechuguilla en general, pero particularmente sobre los métodos, frecuencias e intensidades de recolección. Además, se requiere más investigación relacionada con los usos no tradicionales de los subproductos de la lechuguilla, como es la obtención de insecticidas naturales. Finalmente, cabe proponerse utilizar la planta de lechuguilla como modelo para estudios bioquímico-fisiológicos relacionados con los procesos de tolerancia a sequía, temperaturas extremas u otros, así como con los procesos fotosintéticos MAC-C ${ }_{3}$.

\section{Conclusiones}

El aprovechamiento óptimo y persistente de un recurso natural renovable como la lechuguilla, requiere que se conozca con detalle tanto la especie como el ambiente en que se desarrolla. El ambiente de la lechuguilla ha sido evaluado y caracterizado ampliamente, y en cierta medida se conoce la fenología y morfología de esta especie; sin embargo, sus características fisiológicas y bioquímicas se conocen sólo incipientemente, y se requiere identificar y caracterizar las variantes y su calidad de la fibra. Es importante buscar y evaluar otros usos de la lechuguilla y evaluar los métodos tradicionales de aprovechamiento para fibra.

El estudio de la estructura y dinámica de las poblaciones de lechuguilla con distinto grado de aprovechamiento, así como el efecto de otras formas de uso de las comunidades con lechuguilla es indispensable para fundamentar sistemas persistentes de aprovechamiento.

\section{Literatura citada}

Aguilar C.A. y Zolla C. 1982. Plantas tóxicas de México. Instituto Mexicano del Seguro Social. México, D. F. 271 p.

Aguirre R.J.R. 1983. Enfoques para el estudio de las actividades agrícolas en le altiplano potosino-zacatecano. En: J. T. Molina G. (Ed.) Recursos agrícolas de zonas áridas y semiáridas de México. Colegio de Postgraduados. Chapingo, Méx. pp. 105-115.

Aldrete M.E. 1981. Estudio ecológico de los agostaderos del noreste del estado de Zacatecas. Tesis profesional. Departamento de Zootecnia. Universidad Autónoma Chapingo. Chapingo, Méx. 285 p.

Aldrete M.E. y Aguirre R. J.R. 1982. Diferenciación de sitios y condición de agostaderos en el noreste del estado de Zacatecas. Chapingo 35-36:53-58.

Bailey L.H. y Bailey E.Z. 1976. Hortus third. McMillan. New York, USA. 1290 p.

Belmares H., Barrera A., Castillo E. y Monjarás M.. 1985. El uso de fibras naturales duras como material de contrucción. Estado de avance. En: Cruz C., del Castillo L., Robert M. y Ondarza R.N. Biología y aprovechamiento integral del henequén y otros agaves. Centro de Investigaciones Ecológicas del Sureste. San Cristobal de las Casas, Chis. pp. 231-242.

Belmares H., Castillo J.E., Barrera A. 1979a. Natural hard fibers of the North American continent. Statistical correlations of physical and mechanical properties of lechuguilla (Agave lechuguilla) fibers. Textile Research Journal 49:619-622.

Belmares H., Barrera A., E. Castillo, M. Monjarás, M. Tristán. 1979b. Industrialización de los recursos vegetales de las zonas áridas y semiáridas de América del Norte. Fibras vegetales duras. Interciencia 4(6):320-325.

Berlanga R.C.A., González L.L.A. y Franco L.H. 1992a. Metodología para la evaluación y manejo de lechuguilla en condiciones naturales. Folleto Técnico Núm. 1. Instituto Nacional de Investigaciones Forestales y Agropecuarias, SARH. Saltillo, Coah. 21 p.

Berlanga R.C.A., García V.M. y González L.L.A. 1992b. Técnicas para el establecimiento y manejo de una plantación de lechuguilla. Folleto Divulgativo Núm. 1. Instituto Nacional de Investigaciones Forestales y Agropecuarias, SARH. Saltillo, Coah. 8 p.

Castro-Franco J., García-Alvarado S. y Galán-Wong L.J. 1998. An alternative bioinsecticide formulation to encapsulate Bacillus thuringiensis delta toxin and extracts of Agave lechuguilla Torr. for the control of Spodoptera frugiperda Smith. Phyton 62: (1-2):71-77.

Correll D.S. y Jhonston M.C. 1970. Manual of the vascular plants of Texas. Texas Research Foundation. Renner, Texas. USA. $1881 \mathrm{p}$.

Crane H.R. y Griffin J.B. 1958. University of Michigan radiocarbon dates III. Science 128:117-123. 
de la Garza de la P.F. 1985. Comercialización y costos de producción del ixtle de lechuguilla. Boletín Divulgativo Núm. 73. Instituto Nacional de Investigaciones Forestales, SARH. México, D. F. 37 p.

Dewey L.H. 1965. Fibras vegetales y su producción en América. $3^{3}$ Ed. Agencia para el Desarrollo Internacional. México, D. F. 97 p.

Eguiarte L.V. y Silva Montellano A. 2000. Evolución de la familia Agavaceae: Filogenia, ecología evolutiva de la reproducción y genética de poblaciones. Boletín de la Sociedada Botánica de México 66:131-151.

Eickmeier W.G. y Adams M.S.. 1978. Gas exchange in Agave lecheguilla Torr. (Agavaceae) and its ecological implications. The Southwestern Naturalist 23:473-486.

Escobar R. s.f. Lechuguilla. En: R. Escobar. Enciclopedia agricola y de conocimientos afines. Tomo II. s.p.i. pp 542545.

Flores F.J.D. y Perales G.J.M. 1989. Primer reporte nacional de las plagas asociadas a la lechuguilla Agave lechuguilla Torrey. Agraria 5: 57-73.

Flores M.S. 1986. Estudio citogenético de Agave crassispina Trel. y Agave lechuguilla Torr. en el municipio de Pinos, Zacatecas y en el municipio de Real de Catorce, San Luis Potosí, México. Tesis profesional. Escuela Nacional de Estudios Profesionales, unam. Los Reyes Iztacala, Méx. $124 \mathrm{p}$.

Font Quer P. 1953. Diccionario de botánica. Labor. Barcelona, España. 1244 p.

Freeman C.E. 1973. Some germination responses of lechuguilla (Agave lecheguilla: Agavaceae). Southwestern Naturalist. 18: 125-134.

Freeman C.E. y Reid W.H. 1983. Nectar sugar composition in some species of Agave (Agavaceae). Madroño 30:153158.

Freeman C.E. y Reid W.H. 1985. Aspects of the reproductive biology of Agave lechuguilla Torr. Desert Plants 7:7580.

Freeman C.E., Tiffany R.S., Reid W.H. 1977. Germination responses of Agave lechuguilla, A. parryi and Fouquieria splendens. Southwestern Naturalist 22: 195-204

García de F.A. y de Sicilia A. 1984. El mercado mundial de las fibras duras. Centro de Investigación Científica de Yucatán. Mérida, Yuc. 75 p.

García M. A. y Galván V. R. 1995. Riqueza de las familias Agavaceae y Nolinaceae en México. Boletín de la Sociedad Botánica de México. 56:7-24.

Gentry H. S. 1982. Agaves of continental North America. The University of Arizona Press. Tucson, Arizona. USA. 670 p.

Gómez G.A. 1965. Distribución geográfica y ecología de las plantas tóxicas que afectan al ganado en la zona árida del estado de San Luis Potosí, México. Proceedings of the IX International Grassland Congress. Sao Paulo, Brazil. pp 1267-1270.

González C. A. y Scheffey W. J. A. 1964. Los recursos es- pontáneos y su economía. En: E. Beltrán (Ed.). Las zonas áridas del centro y norte de México. Instituto Mexicano de Recursos Naturales Renovables. México, D. F. pp. 38-71.

Grove A. R. 1941. Morphological study of Agave lechuguilla. Botanical Gazette. 103 (354-365.

Juárez P.M.A., Reyes A.J.A. y Andrade A.J.A. 1996. Flora útil de tres tipos de matorral en el altiplano potosino-zacatecano. Geografia Agricola 22-23:23-37.

Kirby R. H. 1950. Brush-making fibers. Economic Botany 4:243252.

Magallán H.E. F. 1998. Las agaváceas de Querétaro. Tesis profesional. Facultad de Ciencias Naturales. Universidad Autónoma de Querétaro. 155 p.

Márquez A., Cazaurang N., González I., Colunga G-M.P. 1996. Cellulose extraction from Agave lechuguilla fibers. Economic Botany 50:465-468.

Marroquín J.S., Borja L.G., Velázquez C.R., de la Cruz J.A. 1981. Estudio ecológico dasonómico de las zonas áridas del norte de México. Publicación Especial Núm. 2. $2^{\mathrm{a}}$ Ed. Instituto Nacional de Investigaciones Forestales. México, D. F. 166 p.

Martínez M. 1936. Plantas útiles de México. Ediciones Botas. México D. F. 400 p.

Martínez M. 1979. Catálogo de nombres vulgares y científicos de plantas mexicanas. Fondo de Cultura Económica. México, D.F. 1247 p.

McCleary J.A. 1955. Lechuguilla or lecheguilla, a nomenclatural problem. Saguaroland Bulletin 9:80-81.

McLaughlin, S.P. y S.M. Schuck 1985. Fiber properties of several species of Agavaceae from southwestern United States and Northern Mexico. Economic Botany 45:480-486.

Miranda F. y Hernández X.E. 1963. Los tipos de vegetación de México y su clasificación. Boletín de la Sociedad Botánica de México 28:29-179.

Montúfar L.A. 1987. Estudio polínico y etnobotánico del Bolsón de Mapimí. INAH, SEP. México, D.F. 53 p.

Morales B.S. 1988. Análisis dimensional en lechuguilla (Agave lecheguilla Torr.) en "La Sauceda", Ramos Arizpe, Coah. Tesis profesional. Universidad Autónoma Agraria Antonio Narro. Saltillo, Coahuila. 56 p.

Nieto P.C. 1983. La lechuguilla. InIREb Informa. Núm. 63. Instituto Nacional de Investigación sobre Recursos Bióticos. Xalapa, Ver. 3 p.

Nobel P.S. 1998. Los incomparables agaves y cactos. Trillas. México, D. F. 211 p.

Nobel P.S. y Quero E. 1986. Environmental productivity indices for a Chihuahuan Desert CAM plant, Agave lechuguilla. Ecology 67:1-11.

Nobel. P.S. 1988. Environmental biology of agaves and cacti. Cambridge University Press. New York. USA. 270 p.

Orozco L.M., NavaC. R., MedinaT.J.G. y de la Cruz. J.A. 1977. Investigación silvoagropecuaria de las zonas áridas de México, campo experimental Noria de Guadalupe. Monografía Técnico-Cientifica, UAAAN 3:182-265. 
Pennington, C. W. 1957. Tarahumar fish stupefaction plants. Economic Botany. 12:95-102.

Quero E., Nobel P.S. 1987. Predictions of field productivity for Agave lechuguilla. Journal of Applied Ecology 24:10531062.

Ramírez L.A. 1932. Agaves textiles de México. Folletos de Divulgación Científica del Instituto de Biologia 12: 3-22.

Reyes A.J.A. 1992. Estudio florístico de la sierra de Monte Grande, municipio de Charcas, S.L.P. México. Tesis de maestría. Colegio de Postgraduados. Centro de Botánica. Chapingo, Méx. 212 p.

Reyes Agüero. J.A., González Medrano F., García PJ.D. 1996. Flora vascular de la sierra Monte Grande, municipio de Charcas, San Luis Potosí, México. Boletín de la Sociedad Botánica de México 58:31-42.

Reyes A. J. A. y Aguirre R. J. R. 1999. Fitogeografía de la sierra Monte Grande, Charcas, San Luis Potosí, México. Caldasia 21:50-69.

Rodríguez L. F. 1985. Les chihimeques.CEMCA. México, D. F. 239 p.

Rzedowski J. 1964. Botánica económica. En: Beltrán E. (Ed.) Las zonas áridas del centro y noreste de México y el aprovechamiento de sus recursos. Instituto Mexicano de Recursos Naturales Renovables. México, D. F. pp. 135-152.

Rzedowski J. 1965. Vegetación del estado de San Luis Potosí. Acta Cientifica Potosina 5: 3-291.
Rzedowski J. 1978. La vegetación de México. Limusa. México, D. F. 431 p.

Santamaría F.J. 1992. Diccionario de mejicanismos. $5^{\mathrm{a}} \mathrm{Ed}$. Porrúa. México, D. F. 1207 p.

Sheldon S. 1980. Ethnobotany of Agave lecheguilla and Yucca carnerosana in Mexico's Zona Ixtlera. Economic Botany 34:376-390.

Silva M.A., A. Cardonal y Eguiarte L.E.. 1998. Ecología evolutiva de Agave lecheguilla Torr. en un gradiente latitudinal. Memoria del VII Congreso Latinoamericano de Botánica y XIV Congreso Mexicano de Botánica. México, D. F. p. 330.

Simeón R. 1977. Diccionario de la lengua náhuatl o mexicana. Siglo Veintiuno. México, D. F. 783 p.

Standley P.C. 1920-1926. Trees and shrubs of Mexico. US Nat. Mus. Contr. 23:1-1721

Valdés C.M. 1995. La gente del mezquite.CIESAS-INI. México, D. F. 279 p.

Wall M.E., Warnock B.H. y Willaman J.J.. 1962. Steroidal sapogenins. LXVIII. Their ocurrence in Agave lechuguilla. Economic Botany 16:266-269. IB

Zapién B.M. 1981. Evaluación de la producción de ixtle de lechuguilla en cuatro sitios diferentes. En: Anónimo. Primera Reunión Nacional sobre Ecología, Manejo y Domesticación de las Plantas Útiles del Desierto. Publicación Especial Núm. 31. Instituto Nacional de Investigaciones Forestales, SARH. México, D. F. pp. 385-389. 